\title{
ON A CONJECTURE OF SCHINZEL AND ZASSENHAUS
}

\section{YILUN SHANG}

Abstract. A. Schinzel and $\mathrm{H}$. Zassenhaus had the following conjecture regarding algebraic integers: If $\alpha \neq 0$ is an algebraic integer of degree $n$ which is not a root of unity, then there exists a constant $c>0$ such that

$$
|\bar{\alpha}| \geqslant 1+\frac{c}{n}
$$

where $|\bar{\alpha}|=\max _{1 \leqslant i \leqslant n}\left|\alpha_{i}\right|, \alpha_{1}=\alpha$ and $\alpha_{2}, \cdots, \alpha_{n}$ are the conjugates of $\alpha$.

We give some partial solutions to this conjecture in this paper via spectral properties.

Mathematics subject classification (2010): 15A42, 11 R04.

Keywords and phrases: algebraic integer, eigenvalue, conjugate, root of unity.

\section{REFERENCES}

[1] P. E. Blanksby, H. L. Montgomery, Algebraic integers near the unit circle, Acta. Arith., 18(1971) 355-369.

[2] D. W. Boyd, The maximal modulus of an algebraic integer, Math. Comp., 45(1985) 243-249.

[3] D. C. Cantor, E. G. Straus, On a conjecture of D. H. Lehmer, Math. Comp., 42(1982) 97-100.

[4] E. Dobrowols Ki, On the maximal modulus of conjugates of an algebraic integer, Bull. Acad. Polon. Sci. Ser. Sci. Math. Astronom. Phys., 26(1978) 291-292.

[5] E. DobRowolski, On a question of Lehmer and the number of irreducible factors of a polynomial, Acta. Arith., 34(1979) 391-401.

[6] A. DubickAs, On a conjecture of A. Schinzel and H. Zassenhaus, Acta. Arith., 63(1993) 15-20.

[7] D. S. Dummit, R.’ M. Foote, Abstract Algebra, Wiley, New York, 2004.

[8] A. Grytczuk, M. SzaŁkows ki, Spectral properties of some matrices, Acta. Acad. Pead. Agriensis Sectio Math., 20(1991) 43-50.

[9] A. GRYTCZUK, I. KURZYDŁO, On some application of the spectral properties of the matrices, Notes Number Theory Discrete Math., 17(2011) 12-17.

[10] D. H. LeHMER, Factorization of certain cyclotomic functions, Ann. Math., 34(1933) 461-479.

[11] R. Louboutin, Sur la mesure de Mahler d'un nombre algébrique, C. R. Acad. Sci. Paris, 296(1983) 707-708.

[12] E. M. Matveev, On the cardinality of algebraic integers, Mat. Zametki, 49(1991) 152-154.

[13] W. NAR KIEWICZ, Elementary and Analytic Theory of Algebraic Numbers, Polish Scientific Publishers PWN, Warszawa, 1990.

[14] G. RHIn, Q. Wu, On the smallest value of the maximal modulus of an algebraic integer, Math. Comp., 76(2007) 1025-1038.

[15] A. Schinzel, H. Zassenhaus, A refinement of two theorems of Kronecker, Michigan Math. J., 12(1965) 81-85.

[16] C. L. SteWART, Algebraic integers whose conjugates lie near the unit circle, Bull. Soc. Math. France, 196(1978) 169-176. 